ARTICLE

Received 16 Aug 2012 | Accepted 7 Jan 2013 | Published 12 Feb $2013 \quad$ DOl: 10.1038/ncomms2469

\title{
A subset of Drosophila Myc sites remain associated with mitotic chromosomes colocalized with insulator proteins
}

\author{
Jingping Yang ${ }^{1}$, Elizabeth Sung ${ }^{1}$, Paul G. Donlin-Asp ${ }^{1} \&$ Victor G. Corces ${ }^{1}$
}

Myc has been characterized as a transcription factor that activates expression of genes involved in pluripotency and cancer, and as a component of the replication complex. Here we find that Myc is present at promoters and enhancers of Drosophila melanogaster genes during interphase. Myc colocalizes with Orc2, which is part of the prereplication complex, during G1. As is the case in mammals, Myc associates preferentially with paused genes, suggesting that it may also be involved in the release of RNA polymerase II from the promoter-proximal pausing in Drosophila. Interestingly, about $40 \%$ of Myc sites present in interphase persists during mitosis. None of the Myc mitotic sites correspond to enhancers, and only some correspond to promoters. The rest of the mitotic Myc sites overlap with binding sites for multiple insulator proteins that are also maintained in mitosis. These results suggest alternative mechanisms to explain the role of Myc in pluripotency and cancer.

\footnotetext{
${ }^{1}$ Department of Biology, Emory University, 1510 Clifton Road NE, Atlanta, GA 30322, USA. Correspondence and requests for materials should be addressed to V.G.C. (email: vcorces@emory.edu).
} 
$\mathrm{M}$ yc has been extensively studied as an oncogene that has critical roles in cancer initiation and metastasis of many different types of tumours ${ }^{1,2}$. Myc is a sequence-specific DNA-binding protein ${ }^{3}$, which can bind to both the canonical $\left(5^{\prime}\right.$-CACGTG-3' or $5^{\prime}$-CATGTG- $\left.3^{\prime}\right)$ and non-canonical $5^{\prime}$-CANNTG-3' E-box sequences ${ }^{4}$. Although Myc has been found to regulate various cellular processes, including cell growth, cell proliferation and cell differentiation, the mechanisms by which it elicits neoplastic transformation are not well understood.

Myc is a basic helix-loop-helix leucine zipper transcription factor that regulates the expression of protein-coding genes and microRNAs ${ }^{5-8}$. Genomic searches for Myc target genes have uncovered a role for this protein in the regulation of hundreds of genes involved in cell cycle progression, differentiation, apoptosis, DNA repair, angiogenesis, chromosome instability and ribosome biogenesis ${ }^{1,9,10}$. Results suggest that Myc may regulate expression of these genes, at least in part, by interacting with positive transcription elongation factor-b to release RNA polymerase II (RNAPII) from the promoter-proximal pausing, and bringing about productive elongation ${ }^{11-13}$. Most genome-wide studies of Myc in mammalian cells have focused on its presence at promoter regions ${ }^{14-16}$. However, Myc is also found in non-promoter sequences. For example, Myc is enriched in the first intron of genes, and about $10 \%$ of Myc sites are present in intergenic regions ( $>100 \mathrm{~kb}$ from genes) in human $\mathrm{B}$ cells ${ }^{17}$. In mouse fibroblasts, $30.4 \%$ of Myc sites are intergenic ( $>1 \mathrm{~kb}$ from genes) and $22.4 \%$ are intragenic (between $1 \mathrm{~kb}$ downstream of the transcription start site (TSS) and the $3^{\prime}$-end) ${ }^{18}$. These non-promoter Myc sites may function as transcriptional regulatory elements, such as enhancers, but their role has not been studied in detail.

In addition to its role in transcription of genes encoding proteins involved in DNA replication, Myc may also regulate this process directly. Cells overexpressing Myc become polyploidy but do not enter mitosis ${ }^{19}$. In Drosophila, Myc is required for endoreplication $^{20,21}$, and it has been suggested that the role of Myc in replication is independent of transcription ${ }^{22}$. In human cells, the Myc protein interacts with the prereplication complex and it has been shown to be required for recruitment of $\mathrm{Mcm}$ proteins at specific $l o i^{22,23}$, but whether this is a general phenomenon, and Myc colocalizes genome-wide with the origin recognition complex, has not been investigated. Myc controls a variety of cellular processes required for cell differentiation and is essential for cellular reprogramming to induce pluripotency and stem cell renewal ${ }^{24-26}$. The role of Myc in these cellular processes may be a consequence of its effects on gene expression at the local level, but other evidence suggests that Myc can also affect chromatin more globally ${ }^{27}$. An important property of Myc that has been largely ignored when considering potential mechanisms by which this protein can affect gene expression is that it remains bound to DNA during mitosis ${ }^{28,29}$, raising the question of whether some of the functions ascribed to this protein are actually a consequence of its presence in mitotic chromosomes. However, the location and function of Myc in mitotic cells has not been explored.

Here we examine the distribution of Myc during interphase and mitosis in Drosophila Kc cells. We find that Myc colocalizes extensively with Orc2 during interphase, supporting a generalized role for Myc in the prereplication complex. In addition to promoters, Myc is also present at enhancers of the Drosophila genes. Interestingly, only a specific subset of interphase Myc sites remain in mitotic chromosomes. Mitotic Myc sites include a fraction of promoter regions and aligned insulators, where several insulator proteins colocalize within a 300 -bp region. These results suggest that Myc may have an as of yet unappreciated role in the maintenance of chromosome structure and epigenetic information during the cell cycle, which may explain some of its effects in tumorigenesis and pluripotency.

\section{Results}

Myc is present at the promoters of paused genes. To study changes in the distribution of Myc, we performed chromatin immunoprecipitation followed by deep sequencing (ChIP-seq) in Drosophila melanogaster Kc cells, using an antibody against this protein. To distinguish possible roles of Myc in mitosis versus other stages of the cell cycle, we partially synchronized the cells, labelled them with antibodies to H3S10ph or Lamin Dm0, and separated the mitotic and interphase populations using fluorescence-activated cell sorting. Results of ChIP-seq experiments were quantitated and confirmed at three different genomic locations by ChIP-quantitative PCR (Supplementary Fig. S1). To determine whether the role of Myc in transcription and replication is conserved in Drosophila, we first mapped the binding sites for this protein in interphase when the cells are undergoing a process of biomass accumulation and preparing for the next cell cycle. We identified $\sim 4,000$ Myc-binding sites across the genome of interphase cells (Supplementary Data 1). Analysis of these data indicates that Myc associates with coding and non-coding genes (Fig. 1a, left and right panels, respectively, and Supplementary Data 2). Myc binds preferentially to the promoter-proximal regions (between TSSs and $-200 \mathrm{bp}$ ). Only $8 \%$ of Myc sites fall in exons, including $5^{\prime}$ - and $3^{\prime}$-untranslated regions (UTRs). In addition to the promoter regions, Myc also binds significantly in introns ( $21 \%$ of sites) and intergenic regions ( $17 \%$ of sites; Fig. $1 \mathrm{~b})$.

Myc has been extensively characterized as a transcription factor in mammals. In Drosophila, 53\% of Myc sites are located in the promoter regions of genes and $4 \%$ in the $5^{\prime}$-UTRs (Fig. 1b). These sites of Myc associate with genes involved in ribosomal RNA synthesis, cell cycle and development (Supplementary Data 2). To understand how Myc regulates adjacent genes, we examined their expression status. In general, Myc associates with genes that also have RNAPII at the TSS, suggesting they have undergone transcription initiation. However, the difference in RNAPII levels between the promoter region and the gene body is larger for genes with Myc than for genes without Myc (Fig. 1a). These observations suggest that Myc-associated genes have high pausing indexes. In mammalian cells, Myc has a role in the release of RNAPII from promoter-proximal pausing ${ }^{13}$. We therefore examined whether Drosophila Myc is also associated with paused genes by determining the pausing index, which is a measure of the difference in RNAPII levels between the promoter and gene body. The results indicate that Myc preferentially associates with paused genes in Drosophila. More Myc-associated genes show high pausing index than the average genome level. Around $41 \%$ of Mycassociated genes show pausing indexes higher than 1 , whereas only around $16 \%$ of all genes show a pausing index larger than 1 (Kolmogorov-Smirnov (K-S) test, $P<2 \times 10^{-16}$; Fig. 1c). Although Myc-associated genes show high pausing indexes, they are also highly expressed. About $50 \%$ of Myc-associated genes belong to the group with the highest transcription levels (Fig. 1d), suggesting that RNAPII in Myc-associated paused genes is quickly released into productive elongation.

The role of $\mathrm{Myc}$ at non-promoter regions. As previously observed in mammalian cells ${ }^{17,18}$, Drosophila Myc also binds to non-promoter regions of coding genes (Fig. 1b). These intergenic or intronic Myc sites are not located in the promoter regions of non-coding genes. Out of 1,144 intergenic or intronic Myc sites, only 31 are found adjacent to non-coding RNA genes. Genomewide studies of Myc distribution have been carried out in different types of mammalian cells, but no detailed analysis of the function of these non-promoter sites is available. We therefore examined the distribution of histone modifications and RNAPII enrichment at these non-promoter Myc sites. Results indicate that 
a

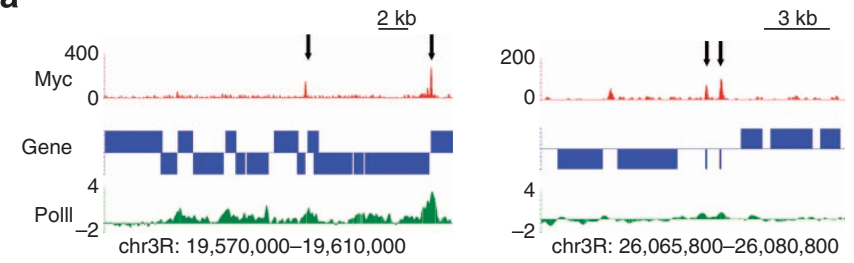

b

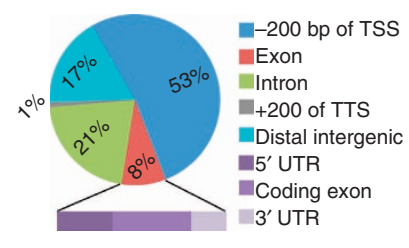

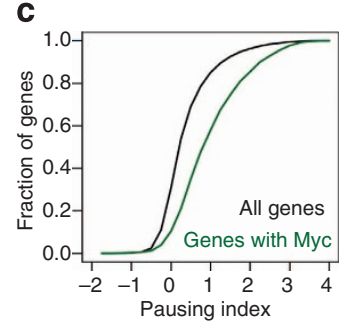

d

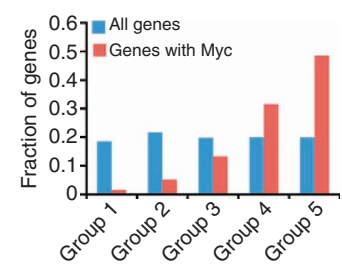

Figure 1 | Characteristics of Myc-associated genes. (a) Examples of Myc-associated genes. The signal for Myc is represented as the number of raw reads from ChIP-seq, and the signal for RNAPII is represented by the log2 enrichment from ChIP-chip. On the gene track, the genes above the line are transcribed from the plus strand and the genes below the line are transcribed from the minus strand. The two arrows on the left point to two protein-coding genes associated with Myc that also show high accumulation of RNAPII at the TSS. The two arrows on the right point to two non-protein-coding microRNA genes associated with Myc. (b) Genome-wide distribution of Myc-binding sites with respect to various gene landmarks. Distal intergenic region means regions that are at least 200 bp away from genes. (c) Cumulative curve of pausing index for all coding genes in the genome (black) or coding genes associated with Myc (green). The two distributions are significantly different (K-S test, $P<2 \times 10^{-16}$ ). (d) Distribution of expression levels of Myc target genes. All genes in the genome were sorted according to their expression score and binned into five groups (Group 1 with the lowest expression and Group 5 with the highest expression). Myc target genes were assigned to one of the groups if their expression scores fall into the range of expression levels for that group.

a subset of these Myc sites show chromatin signatures characteristic of enhancers (Fig. 2a,b). Myc sites at promoter regions are enriched in $\mathrm{H} 3 \mathrm{~K} 4 \mathrm{me} 3$ and RNAPII. In contrast, a subset of Myc sites at non-promoter regions show H3K4me1, whereas H3K4me3 and RNAPII are absent (Fig. 2b). Enrichment in $\mathrm{H} 3 \mathrm{~K} 4 \mathrm{me} 1$ without $\mathrm{H} 3 \mathrm{~K} 4 \mathrm{me} 3$ is characteristic of enhancers ${ }^{30}$. These Myc sites also contain $\mathrm{H} 3 \mathrm{~K} 27 \mathrm{ac}$, indicating they correspond to active enhancers (Fig. 2b). We have previously identified all enhancers in Drosophila Kc cells ${ }^{31}$, and we used this information here to compare enhancers with and without Myc. The results suggest that most active enhancers containing $\mathrm{H} 3 \mathrm{~K} 27 \mathrm{ac}$ also have Myc, whereas inactive enhancers lacking H3K27ac are depleted of Myc (Fig. 2c). In fact, if we consider the subset of Myc-containing enhancers located in introns to be able to assign enhancers to their target genes, Myc-containing enhancers are present in the most actively transcribed genes (Fig. 2d). Thus, a subset of non-promoter Myc sites appears to be present at active enhancers that regulate highly transcribed genes. However, not all the non-promoter Myc sites show enhancer-like chromatin features. A second subset of non-promoter Myc sites (denoted with a question mark in Fig. 2b) is depleted of $\mathrm{H} 3 \mathrm{~K} 4 \mathrm{me} 1, \mathrm{H} 3 \mathrm{~K} 4 \mathrm{me} 3$ and H3K27ac, and therefore, it does not correspond to enhancers or promoters.

Myc associates with Orc2 genome wide in D. melanogaster. In mammalian cells, the Myc protein interacts with the prereplication complex, and it has been found at specific DNA replication origins with origin recognition complex (Orc) proteins ${ }^{22}$. To test whether this is also the case in Drosophila, and some of the sites with unknown function in Fig. $2 \mathrm{~b}$ correspond to replication origins, we compared the binding profiles of Myc with the Orc2 component of the complex. The signals for the two proteins show significant overlap in specific regions of the genome (Fig. 3a). We then examined genome-wide correlations between the two proteins, using heatmaps to visualize the information. The results indicate that Orc2 is present at most Myc sites in the genome and vice versa (Fig. 3b). Thus, Myc colocalizes with Orc2 genomewide in Drosophila cells at both promoter and non-promoter regions.

A subset of Myc sites is bound to chromosomes during mitosis Myc has been shown to be present in mitotic chromosomes ${ }^{28,29}$, but its specific distribution in chromatin during mitosis has never been analysed. The presence of Myc in mitotic chromosomes may be critical for its role in transcription. To gain further insights into mechanisms by which Myc affects gene expression, we mapped Myc-binding sites in mitotic cells and compared the distribution of this protein between interphase and mitosis. The results indicate that all Myc mitotic sites are also occupied by this protein during interphase, but not all Myc sites in interphase are retained in mitosis (Fig. 4a). Myc sites in the genome can therefore be classified as interphase-specific (Class I) or common to mitosis and interphase (Class II). We will refer to this second group as mitotic sites, although they are also present in interphase (Fig. 4a). In interphase, the average enrichment of Myc at Class II sites is only about half of the average enrichment at Class I sites, but the enrichment is significantly higher than background (Fig. 4b, top panel). In mitosis, there is no significant enrichment for Myc at Class I sites (Fig. 4b, bottom panel). The mechanism by which Myc persists at only a subset of interphase sites may depend on the specific recognition sequence present at each class of sites. We therefore examined potential differences in the consensus motif at Class I and Class II sites. E-boxes can be found in about $70 \%$ of Myc sites in either class, but the two classes show different preferences for specific sequences. Myc sites in Class I preferentially contain the canonical E-box (CATGTG/CACGTG). In contrast, Myc sites in Class II sites are depleted of the canonical E-box and, instead, show enrichment for the noncanonical E-box (Fig. 4c). The preference in utilization of each E-box type is significant $\left(\chi^{2}\right.$-test, $\left.P<0.0001\right)$ and may represent the underlying mechanism to select Myc sites that will be maintained during the cell cycle. 
a

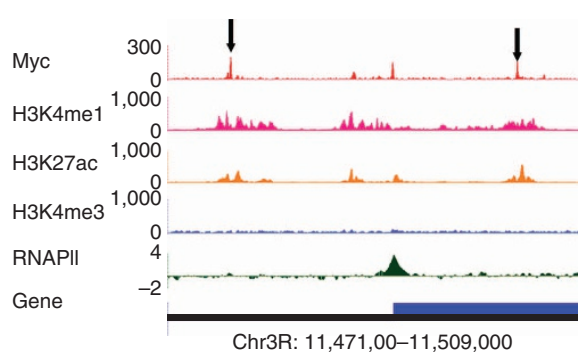

b

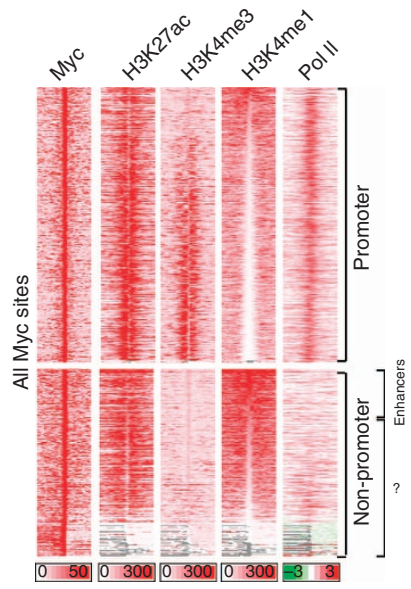

C

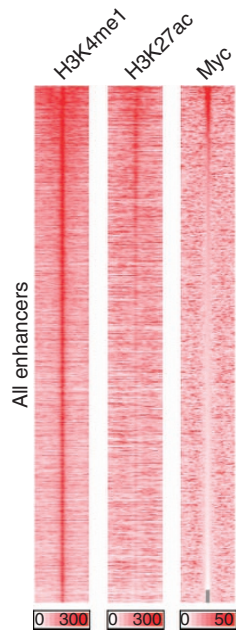

d

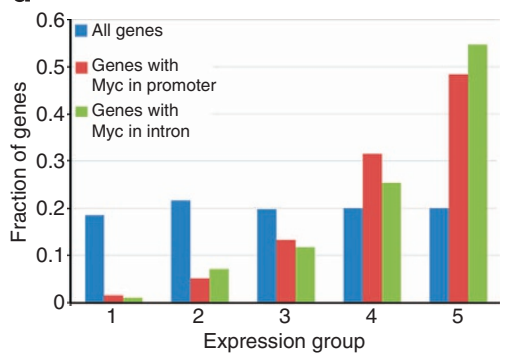

Figure 2 | A subset of Myc sites at non-promoter regions have characteristics of enhancers. (a) Examples of non-promoter Myc sites in the genome. The signals on the tracks of Myc, H3K4me1, H3K27ac and H3K4me3 are represented by the raw reads from ChIP-seq. The signal for RNAPII is represented by the log2 value from ChIP-chip. The arrows represent two Myc sites that display enhancer chromatin signatures (presence of H3K4me1/H3K27ac and absence of H3K4me3). (b) Heatmaps showing the chromatin features at promoter and non-promoter Myc sites. Each panel represents $2 \mathrm{~kb}$ upstream and downstream of the Myc sites. The sites are ordered by signal of H3K4me1. (c) Heatmaps showing chromatin features at all identified enhancers in Kc cells. The sites are ordered by signal of Myc. (d) Fraction of genes containing Myc at the promoter or enhancer expressed at different levels compared with all genes in the genome. Group 1 genes are expressed at low levels and Group 5 genes at the highest levels (see Methods).

Two classes of Myc sites with different roles in transcription. To gain insights into possible functional differences between interphase-specific and mitotic Myc sites, we first performed gene ontology analysis for genes associated with each class. Class I Myc sites are enriched at genes involved in ribosome biogenesis, which is important for biomass accumulation in G1. This is consistent with reports for Myc-regulated genes in interphase cells in mammals 9 . However, genes associated with Class II Myc sites are not enriched for this category (Fig. 5a). In addition to their presence at different target genes, the two groups of Myc sites may also affect gene expression by different mechanisms. In interphase cells, Myc-associated genes generally show higher pausing index than the average gene in the genome. We then parsed genes into two groups based on their association with Class I or Class II Myc sites. The results suggest that genes associated with Class I Myc sites (interphase-specific) still show a high pausing index, whereas genes associated with Class II Myc sites (those also present in mitosis) have slightly but statistically significant lower pausing indexes (K-S test, $P=2 \times 10^{-6}$; Fig. 5b). Class I Myc sites may then be involved in the release of paused RNAPII for productive elongation in interphase cells, whereas Class II Myc sites may have a different regulatory function in transcription that is dependent on the presence of Myc protein in mitotic chromosomes.
Mitotic Myc sites are present at promoters but not at enhancers. To further explore functional differences between the two classes of Myc sites, we clustered all the sites with histone modifications characteristic of enhancers and promoters using $k$-means clustering. The results reveal five clusters of Myc sites (Fig. 5c). Class I Myc sites are present in three different clusters, whereas class II Myc sites associate with two clusters. Class I sites are present at enhancers (Cluster I), promoters (Cluster III) and a cluster lacking either characteristic (Cluster II). Class II sites are present at promoters (Cluster IV) and a cluster of unknown function (Cluster V). Therefore, Myc is present at enhancers only during interphase and persists during mitosis at only a specific subset of all promoters occupied during interphase (Fig. 5c). Interestingly, Myc-associated promoters in both interphase and mitosis appear to cluster in two groups with high or low levels of H3K4me3. In Drosophila, enhancers defined as sequences enriched in H3K27ac and H3K4me1, but lacking H3K4me3, are typically found within intronic regions ${ }^{32}$. Consistent with the clustering results, $55 \%$ of Class I non-promoter Myc sites are in introns, whereas $70 \%$ of Class II non-promoter Myc sites fall in intergenic regions $(P<0.0001$; Fig. $5 \mathrm{~d})$. These results suggest that Class II Myc sites do not function as enhancers and they may have a different role in the genome independent of transcription. The possibility of a different role for Class II sites is supported by 


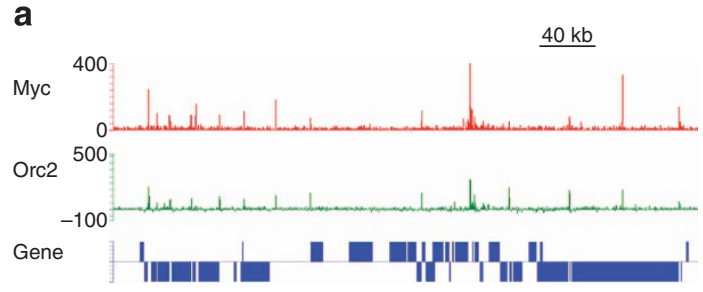

b

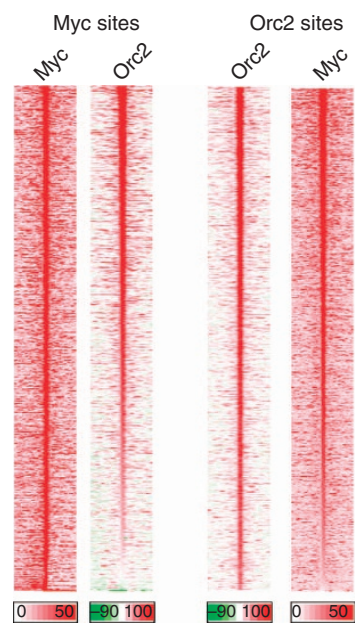

Figure 3 | Myc associates with Orc2 genome-wide. (a) Snapshot of a region of the Drosophila genome showing the distribution of Orc2 sites compared with Myc. The signals represent the number of raw reads from ChIP-seq data sets across the region. (b) Heatmaps of Myc and Orc2 signal at all Myc- and Orc2-binding sites. Each panel represents $2 \mathrm{~kb}$ upstream and downstream of the anchor sites. The two panels on the left are the signals at all Myc-binding sites in Kc cells discovered in this study ordered by Orc2 signal intensity. The two panels on the right are the signals at all Orc2-binding sites obtained from modENCODE ordered by Myc signal. the observation that these sites are further apart from each other compared with Class I sites (Fig. 5e).

Myc sites of unknown function associate with insulators. A subset of Myc sites in both Class I and Class II are not present at either enhancers or promoters. A third type of regulatory sequences found in eukaryotic cells is represented by insulators. To test whether the Myc sites of unknown function are present at insulators, we parsed ChIP-seq data sets of Drosophila insulator proteins BEAF-32, dCTCF, Su(Hw), GAF and CP190, with the clusters shown in Fig. $5 c$. The results show a dramatic difference between Class I and Class II sites (Fig. 6a). Class I sites associate preferentially with GAF, both at enhancers and promoters where this protein has been shown to be present ${ }^{33}$, as well as in Cluster II containing sites not present at these two types of regulatory sequences. A subset of interphase-specific Class I promoter sites present in Cluster III, those containing high levels of $\mathrm{H} 3 \mathrm{~K} 4 \mathrm{me} 3$ and presumably actively transcribed, contain BEAF-32 instead of GAF. Class II sites, on the other hand, associate with insulator proteins other than GAF (Fig. 6a). In particular, all Class II sites, including those in Cluster $\mathrm{V}$, contain all four insulator proteins tested, Su(Hw), BEAF-32, dCTCF and CP190 (Fig. 6a, b).

Myc mitotic sites associate with mitotic insulator sites. The results presented above suggest a strong association between Class II Myc sites and sites of specific insulator proteins from interphase cells. As Class II sites persist during mitosis, we wondered whether insulator proteins also remain at these sites during mitosis. To test this possibility, we mapped the binding of insulator proteins in mitosis and compared the distribution of Class II Myc sites with data sets of insulator protein localization in mitotic chromosomes. The results indicate that Myc overlaps extensively with insulator proteins during mitosis (Fig. 6c). All mitotic Myc sites contain dCTCF and CP190, and a subset also contains BEAF-32. A fourth insulator protein, $\mathrm{Su}(\mathrm{Hw})$, is not present in a

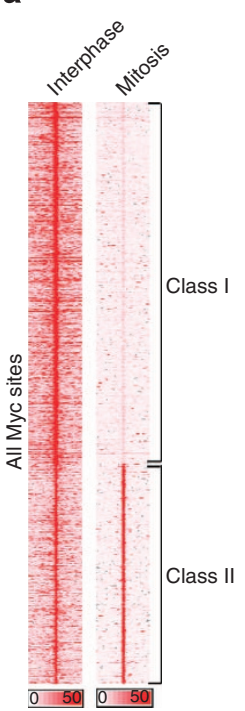

b
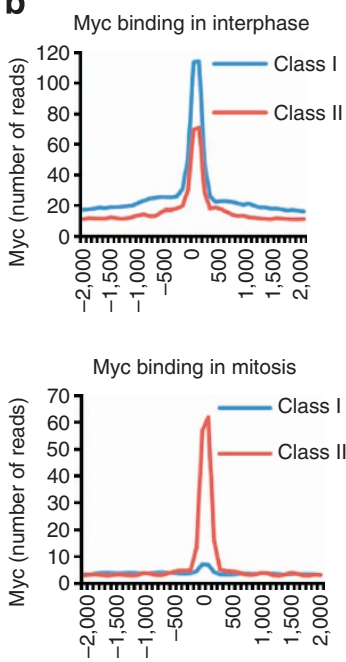

C

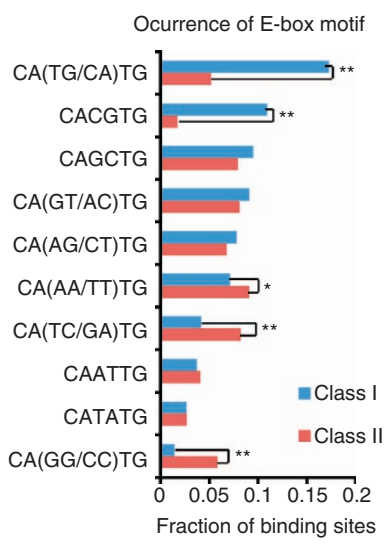

Figure 4 | Properties of Myc sites in interphase and mitotic chromosomes. (a) Heatmap showing signals of Myc in interphase or mitosis at all the Myc site in the genome. The information indicates the existence of two groups of Myc sites in the genome, one is interphase-specific (Class I) and the second one is common to interphase and mitosis (Class II). (b) Binding intensity at Myc sites during interphase or mitosis plotted from the information displayed in panel a. The $x$ axis represents distance from Myc sites and ' $O$ ' is the summit of Myc sites. Negative values indicate upstream and positive values indicate downstream of the Myc sites. (c) Usage of different binding motifs by the Myc protein in interphase (Class I) or mitosis (Class II). The significance of this difference was tested by the $\chi^{2}$-test for each motif $\left({ }^{\star \star} P<0.01,{ }^{\star} P<0.05\right)$. 
a

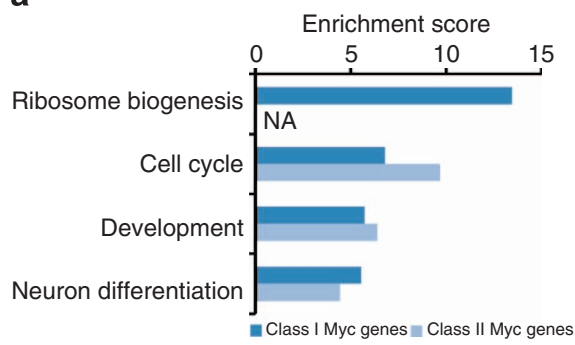

C

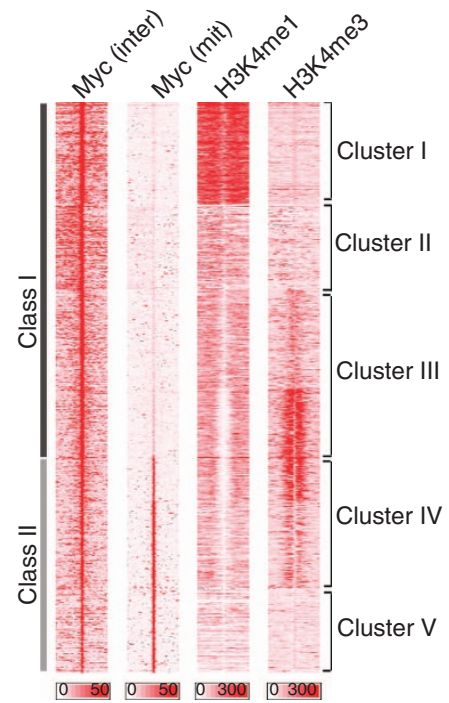

b

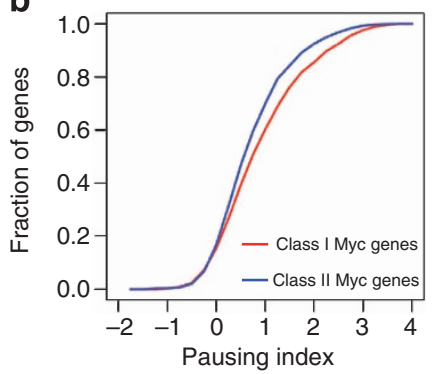

d

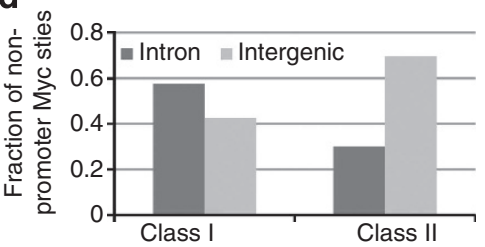

e

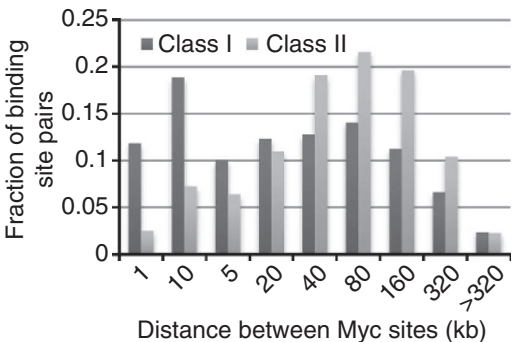

Figure 5 | Myc sites occupied during interphase and mitosis have different characteristics. (a) Gene ontology of protein-coding genes associated with the two classes of Myc sites. (b) Cumulative curve of pausing index for protein-coding genes associated with Class I (red) or Class II (blue) Myc sites. The two distributions are significantly different (K-S test, $P<2 \times 10^{-6}$ ). (c) Heatmaps showing chromatin features of Class I and Class II Myc sites. Each panel represents $2 \mathrm{~kb}$ upstream or downstream of the Myc sites. Clusters were created using Cluster3.0 based on the signal value for the listed features at the Myc sites. (d) Distribution of Class I and Class II non-promoter Myc sites in introns or intergenic regions. (e) Distance between Myc site pairs for Class I and Class II Myc sites.

chromosomes during mitosis (Supplementary Fig. S2). Interestingly, a subset of the sites where Myc and Orc2 colocalize during interphase are sites where Myc persists during mitosis (Fig. 6c).

Mitotic Myc sites localize at borders of topological domains. The role of Myc during mitosis may be local, that is, to mark a subset of promoters or origins of replication for rapid resumption of transcription or assembly of the prereplication complex at the beginning of G1. Alternatively, Myc may have a more global role in chromatin organization. Recent work suggests that eukaryotic chromosomes during interphase are organized into topological domains, characterized by high frequency of interactions, and separated by domain borders ${ }^{34-38}$. These borders are enriched in insulator proteins, which may contribute to the formation of boundaries that separate topological domains. It is possible that some of this organization persist during mitosis, and that insulator proteins contribute to the maintenance of chromosome architecture during the cell cycle. The fact that Myc persists at the same genomic sites as insulator proteins in mitotic chromosomes suggest that it may also be present at domain borders. To test this hypothesis, we compared the distribution of Class I and Class II Myc sites with respect to domain borders previously defined in Drosophila embryonic nuclei ${ }^{38}$. The results suggest that this is indeed the case (Fig. $6 \mathrm{~d}$ ). Class II Myc sites that remain on chromosomes during mitosis are significantly enriched at domain borders, whereas interphase- specific Class II sites are significantly enriched inside domains. These results could be interpreted to suggest that a specific subset of Myc sites may remain bound to chromosomes during mitosis to organize the higher-order structure of chromatin. Alternatively, the presence of Myc at domain borders may be a consequence, rather than a cause, of chromosome organization.

\section{Discussion}

Myc is a basic helix-loop-helix leucine zipper sequence-specific DNA-binding protein that has a crucial role in the regulation of critical cellular processes, such as cell growth, cell division and cell differentiation. Importantly, an increase of Myc levels in the cell leads to oncogenic transformation ${ }^{1}$. The Myc protein is present in most or all proliferating cells of normal tissues, and its expression depends on the existence of mitogenic signals. In addition, expression of Myc is sufficient to induce cell division of most normal cells. As Myc interacts with DNA in a sequencespecific manner, its role in cell proliferation has been explained, based on its ability to control the expression of specific genes by activation or repression of transcription. Interestingly, recent results suggest that the role of Myc in gene expression is not to turn on the transcription of specific genes but rather to amplify the transcriptional output of genes that are already being expressed ${ }^{39,40}$. The effects of $\mathrm{Myc}$ in transcription and replication have been rationalized on the basis of its involvement in the control of promoter-proximal pausing 
a

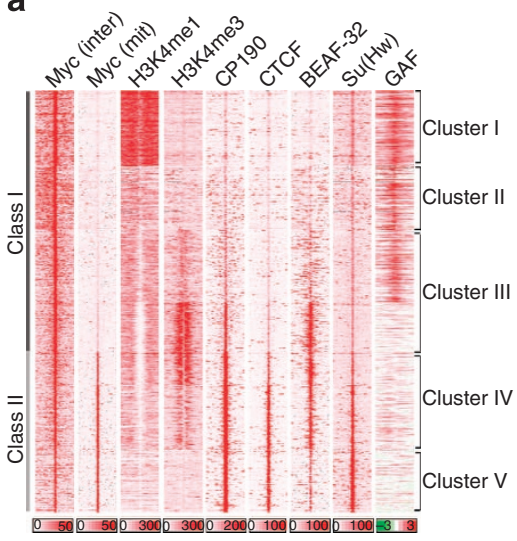

C

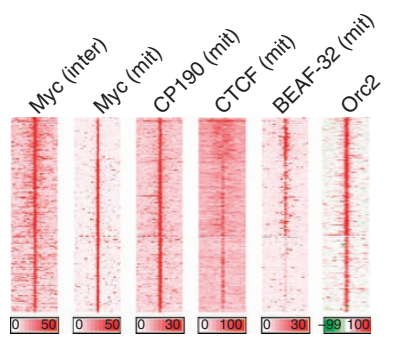

b

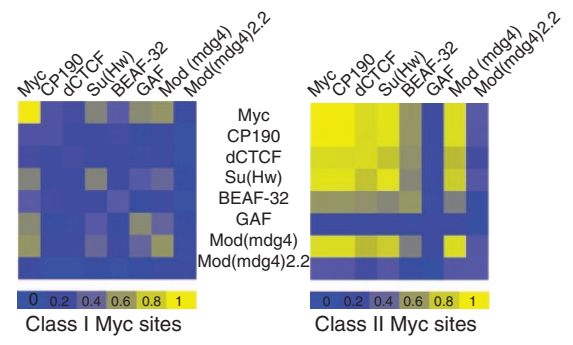

d

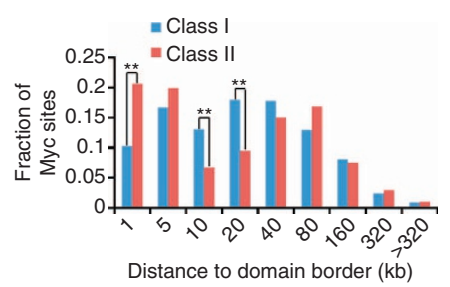

Figure 6 | Myc sites present in mitotic chromosomes associate with insulator proteins. (a) Heatmaps showing chromatin features at Class I and Class II Myc sites. Each panel represents $2 \mathrm{~kb}$ upstream or downstream of the Myc sites. Signals are represented by the number of raw reads from ChIP-seq or enrichment $\log 2$ value from ChIP-chip for the listed proteins or histone modifications. (b) Heatmaps showing the overlap of the listed protein at the two classes of Myc sites. (c) Insulator proteins and Myc persist on chromatin during mitosis at Class II Myc sites. (d) Distribution of Class I and Class II Myc sites with respect to topological domain boundaries. The significance was examined for each distance category by the $\chi^{2}$-test $\left({ }^{\star \star} P<0.01,{ }^{\star} P<0.05\right)$.

of RNAPII and its effects on chromatin structure at the level of histone covalent modifications. Myc can induce $\mathrm{H} 4$ acetylation $^{23,41}$, which correlates with an increase of H4K20me2 and a transient increase of H4K20mel (ref. 41). H4K20mel can function at the crossroad of genome integrity, cell cycle and transcription $^{42}$, and $\mathrm{H} 4 \mathrm{~K} 20 \mathrm{me} 2$ is recognized by Orcl, which is a component of the Orc complex mediating preDNA replication licensing. The bromo adjacent homology domain of Orc1 specifically recognizes $\mathrm{H} 4 \mathrm{~K} 20 \mathrm{me} 2$, a property common to bromo adjacent homology domains present within diverse metazoan Orc1 proteins ${ }^{43}$. The sole enzyme that catalyses H4K20me1 is Setd8 (also known as PR-Set7 or KMT5a), which is an essential mediator of Myc-induced epidermal differentiation. Deletion of Setd8 in Myc-overexpressing skin cells blocks proliferation and differentiation ${ }^{44}$.

Although the ability of Myc to act as a sequence-specific transcription factor and elicit changes in the $10-\mathrm{nm}$ chromatin fibre may account for many of its effects on cell function, the finding of Myc in the proteome of mitotic chromosomes ${ }^{28}$ represents an interesting puzzle. It is possible that Myc persistence on chromatin during mitosis has no relevance to its role in nuclear biology. On the other hand, several aspects of the distribution of Myc in mitotic chromosomes offer tantalizing explanations for some of its effects on transcription and replication. By comparing Myc-binding sites in cells at interphase and mitosis, we find two distinct groups of Myc sites. Class I sites only harbour Myc during interphase, but become devoid of this protein during mitosis. These sites are adjacent to genes involved in ribosome biogenesis, which have been reported to be cell-type and species-independent Myc $\operatorname{targets}^{45}$. In contrast, Class II Myc sites that persist during mitosis associate with genes that have roles in cell cycle or cell differentiation. In mammalian cells, this includes genes important for maintaining pluripotency and reprogramming 45 It is possible that the presence of Myc at these genes during mitosis serves to preserve epigenetic memory of their expression necessary for the maintenance of cell identity.

The striking overlap of Myc and insulator sites during mitosis points to a more complex role for this protein in mitotic chromatin. Insulators have been shown to mediate long-range intra- and interchromosomal interactions ${ }^{46}$. Although the role of some of these interactions may be to regulate enhancer-promoter contacts, the finding of insulators at the boundaries of topological chromosome domains points to a larger and more complex function of these proteins in higher-order chromatin organization. The presence of Myc together with insulator proteins at these sites in mitotic chromosomes may explain some Myc-dependent phenotypes, including its effects on genome integrity. Mouse iPS cell lines induced with Myc show a significantly higher frequency of translocations than those induced without c-Myc ${ }^{47}$. This result is Myc dependent, as deletion of Myc box II reduces the translocation frequency ${ }^{48}$. Myc overexpression also induces telomeric aggregation in the interphase nucleus ${ }^{49}$. These effects of Myc on genomic integrity suggest that Myc may have a role in chromosome higher-order structure that may depend on its presence at insulator sites during interphase and/or mitosis. This conclusion is further supported by the observation that mitotic Myc sites are enriched at the borders of topological chromosome domains, which are also enriched in insulator proteins. Domain boundaries are more accessible to the insertion of transposable elements and allow higher expression of transgenes, suggesting that they represent regions of the genome with more open higher-order chromatin ${ }^{35}$. Together, these observations agree with a model by which insulators organize the chromatin in the interphase nucleus by mediating interactions that create chromosomal domains. 
Transition from interphase to mitosis involves a condensation of the chromatin that nevertheless maintains this organization via the persistence of insulator proteins at domain boundaries. The boundary regions contain more open chromatin that may become accessible to components of the transcription and replication apparatus earlier at the end of M-phase. The maintenance of Myc at these domain boundaries may ensure that adjacent genes are transcribed early at the M/G1 transition, and a subset of replication origins assemble prereplication complexes by recruiting Orc2 and, perhaps, determining replication timing. Additional experiments will be necessary to test this speculative but plausible model.

\section{Methods}

Cell culture and flow cytometry. Drosophila Kc167 cells were grown at $25^{\circ} \mathrm{C}$ in CCM3 media (Hyclone) to a density of $2 \times 10^{6}$. For cell synchronization, the culture was treated with hydroxyurea $\left(1 \mathrm{mg} \mathrm{ml}^{-1}\right.$ in ethanol to a final concentration of $\left.15 \mathrm{ng} \mathrm{ml}^{-1}\right)$ for $16 \mathrm{~h}$, incubated for $8 \mathrm{~h}$ with nocodazole $\left(5 \mathrm{mg} \mathrm{ml}^{-1}\right.$ in dimethylsulphoxide, to a final concentration of $2 \mathrm{ng} \mathrm{ml}^{-1}$ ) and collected. For flow cytometry, cells were fixed for $10 \mathrm{~min}$ in $1 \%$ formaldehyde, blocked in suspension for $30 \mathrm{~min}$ in blocking buffer, incubated overnight with rabbit $\alpha$-H3S10ph at 1:5,000 or mouse $\alpha$-Lamin Dm0 at 1:500, washed $3 \times 15$ min in blocking buffer, and then incubated with secondary antibody Alexa Fluor $488 \alpha$-rabbit at 1:5,000. After a 30 -min incubation in blocking buffer, plus propidium iodide $\left(0.1 \mathrm{mg} \mathrm{ml}^{-1}\right)$, samples were passed several times through a 25 -gauge syringe to reduce clumping and sorted on a FACSAria II cell sorter. Enrichment of the mitotic and interphase cell populations was carried out by visualization of the mitotic marker H3S10ph by immunofluorescence microscopy, showing 97-99\% purity (Supplementary Fig. S2).

ChIP-seq analysis. ChIP was performed with $\sim 4 \times 10^{7}$ cells. Cells were crosslinked with $1 \%$ formaldehyde for $10 \mathrm{~min}$ at room temperature. Nuclear lysates were sonicated to generate 200-1,000 bp DNA fragments. ChIP was then performed with $6 \mu \mathrm{l}$ of Drosophila $\alpha$-Myc antibody (Santa Cruz Biotechnology, sc-28208), $\alpha$-CP190, $\alpha$-CTCF or $\alpha$-BEAF-32 antibodies ${ }^{50,51}$. Libraries were prepared with the Illumina TruSeq DNA Sample Preparation Kit. Fragments in the 200-300 bp range were selected and sequenced in an Illumina HiSeq sequencer at the HudsonAlpha Institute for Biotechnology.

Bioinformatics analyses. Sequences were aligned to Drosophila $\mathrm{dm} 3$ using Bowtie. The output map files were converted to bed format for each chromosome arm, using the VancouverShort package (http://vancouvershortr.sourceforge.net/). Peaks were called using CCAT3.0 (ref. 52), with the enrichment parameter set to 15. Myc-associated genes were defined as genes with Myc-binding sites between $-200 \mathrm{bp}$ and the TSS or in the $5^{\prime}$-UTR region.

In addition to the Drosophila Myc data obtained in this study, we used several data sets obtained from public sources. Orc2 ChIP-seq (modENCODE_2755), RNAPII ChIP-chip (modENCODE_328) and RNA expression in Kc cells (modENCODE_3305) were obtained from modENCODE. ChIP-seq data sets for $\mathrm{H} 3 \mathrm{~K} 4 \mathrm{me} 1, \mathrm{H} 3 \mathrm{~K} 4 \mathrm{me} 3$ and H3K27ac are from GSE36374. ChIP-seq data sets for insulator proteins are from GSE30740, GSE32584 and GSE39664. To build heatmaps, values for each ChIP-seq data set were extracted for the 2,000-bp region around the summit of peaks using custom $\mathrm{R}$ scripts (available upon request), and heatmap graphs were created using TreeView. Clusters in Figs. $5 \mathrm{c}$ and $6 \mathrm{a}$ were created by $k$-means clustering, using Cluster 3.0 based on the mean values of the $300 \mathrm{bp}$ around Myc sites for the samples listed.

The pausing index of genes was calculated using ChIP-chip data sets of RNAPII in Kc cells obtained from modENCODE. RNAPII at TSSs $\left(\mathrm{P}_{\mathrm{TSS}}\right)$ was calculated as the mean enrichment of RNAPII at the 200-bp region around each TSS. RNAPII in the gene body $\left(P_{\text {body }}\right)$ was calculated as the mean enrichment of RNAPII from $+200 \mathrm{bp}$ to the end of the gene. The pausing index is defined as the difference between the $P_{\text {Tss }}$ and $P_{\text {body. }}$. Motif analysis of Myc-binding sites was performed using Myc peak summits, extended $50 \mathrm{bp}$ on either side. The resulting $100 \mathrm{bp}$ sequence for each peak was used to search for E-boxes, using a custom Perl script available upon request. Gene ontology analysis for Myc-associated genes was performed with DAVID (http://david.abcc.ncifcrf.gov). Flybase IDs were used to determine statistically enriched biological process categories on the basis of a background list of all annotated genes in the Drosophila genome. To calculate the different expression groups and the differences in expression levels between these groups, we separated all the annotated genes into five groups with the same number of genes in each group. The expression score was obtained from ModEncode and was created based on the normalized tilling array data from 25 cell lines and 30 developmental stages. Here we use the expression score for Kc cells. The range of the expression score $(\log 10($ score $))$ for each group is as follows: Group 1, $\leq 1.65$; Group 2, 1.65-2.13; Group 3, 2.13-2.44; Group 4, 2.44-2.82 and Group $5,2.82-4.53$.

\section{References}

1. Dang, V. MYC on the path to cancer. Cell 149, 22-35 (2012).

2. Wolfer, A. \& Ramaswamy, S. MYC and metastasis. Cancer Res. 71, 2034-2037 (2011).

3. Blackwell, T., Kretzner, L., Blackwood, E., Eisenman, R. \& Weintraub, H. Sequence-specific DNA binding by the c-Myc protein. Science 250, 1149-1151 (1990).

4. Blackwell, T. K. et al. Binding of myc proteins to canonical and noncanonical DNA sequences. Mol. Cell. Biol. 13, 5216-5224 (1993).

5. O’Donnell, K. A., Wentzel, E. A., Zeller, K. I., Dang, C. V. \& Mendell, J. T. c-Myc-regulated microRNAs modulate E2F1 expression. Nature 435, 839-843 (2005).

6. Aguda, B. D., Kim, Y., Piper-Hunter, M. G., Friedman, A. \& Marsh, C. B. MicroRNA regulation of a cancer network: Consequences of the feedback loops involving miR-17-92, E2F, and Myc. Proc. Natl Acad. Sci. USA 105, 19678-19683 (2008).

7. Lovén, J. et al. MYCN-regulated microRNAs repress estrogen receptor- $\alpha$ (ESR1) expression and neuronal differentiation in human neuroblastoma. Proc. Natl Acad. Sci. USA 107, 1553-1558 (2010).

8. Chang, T.-C. et al. Widespread microRNA repression by Myc contributes to tumorigenesis. Nat. Genet. 40, 43-50 (2008).

9. van Riggelen, J., Yetil, A. \& Felsher, D. W. MYC as a regulator of ribosome biogenesis and protein synthesis. Nat. Rev. Cancer 10, 301-309 (2010).

10. Meyer, N. \& Penn, L. Z. Reflecting on 25 years with MYC. Nat. Rev. Cancer 8, 976-990 (2008).

11. Gargano, B., Amente, S., Majello, B. \& Lania, L. P-TEFb is a crucial co-factor for Myc transactivation. Cell Cycle 6, 2031-2037 (2007).

12. Kanazawa, S., Soucek, L., Evan, G., Okamoto, T. \& Peterlin, B. M. c-Myc recruits $\mathrm{P}-\mathrm{TEFb}$ for transcription, cellular proliferation and apoptosis. Oncogene 22, 5707-5711 (2003).

13. Rahl, P. B. et al. c-Myc regulates transcriptional pause release. Cell 141, 432-445 (2010).

14. Guccione, E. et al. Myc-binding-site recognition in the human genome is determined by chromatin context. Nat. Cell Biol. 8, 764-770 (2006).

15. Kidder, B. L., Yang, J. \& Palmer, S. Stat3 and c-Myc genome-wide promoter occupancy in embryonic stem cells. PLoS ONE 3, e3932 (2008).

16. Li, Z. et al. A global transcriptional regulatory role for c-Myc in Burkitt's lymphoma cells. Proc. Natl Acad. Sci. USA 100, 8164-8169 (2003).

17. Zeller, K. I. et al. Global mapping of c-Myc binding sites and target gene networks in human B cells. Proc. Natl Acad. Sci. USA 103, 17834-17839 (2006).

18. Perna, D. et al. Genome-wide mapping of Myc binding and gene regulation in serum-stimulated fibroblasts. Oncogene 31, 1695-1709 (2012).

19. Li, Q. \& Dang, C. V. c-Myc overexpression uncouples DNA replication from mitosis. Mol. Cell. Biol. 19, 5339-5351 (1999).

20. Pierce, S. B. et al. dMyc is required for larval growth and endoreplication in Drosophila. Development 131, 2317-2327 (2004).

21. Maines, J. Z., Stevens, L. M., Tong, X. \& Stein, D. Drosophila dMyc is required for ovary cell growth and endoreplication. Development 131, 775-786 (2004).

22. Dominguez-Sola, D. et al. Non-transcriptional control of DNA replication by c-Myc. Nature 448, 445-451 (2007).

23. Swarnalatha, M., Singh, A. K. \& Kumar, V. The epigenetic control of E-box and Myc-dependent chromatin modifications regulate the licensing of lamin B2 origin during cell cycle. Nucleic Acids Res. 40, 9021-9035 (2012).

24. Smith, K. N., Singh, A. M. \& Dalton, S. Myc represses primitive endoderm differentiation in pluripotent stem cells. Cell Stem Cell 7, 343-354 (2010).

25. Moumen, M. et al. The proto-oncogene Myc Is essential for mammary stem cell function. Stem Cells 30, 1246-1254 (2012).

26. Varlakhanova, N. V. et al. Myc maintains embryonic stem cell pluripotency and self-renewal. Differentiation 80, 9-19 (2010).

27. Varlakhanova, N. V. \& Knoepfler, P. S. Acting locally and globally: Myc's ever-expanding roles on chromatin. Cancer Res. 69, 7487-7490 (2009).

28. Ohta, S. et al. The protein composition of mitotic chromosomes determined using multiclassifier combinatorial proteomics. Cell 142, 810-821 (2010).

29. O’Donovan, K. J., Diedler, J., Couture, G. C., Fak, J. J. \& Darnell, R. B. The onconeural antigen $\mathrm{cdr} 2$ is a novel $\mathrm{APC} / \mathrm{C}$ target that acts in mitosis to regulate C-Myc target genes in mammalian tumor cells. PLoS ONE 5, e10045 (2010).

30. Heintzman, N. D. et al. Distinct and predictive chromatin signatures of transcriptional promoters and enhancers in the human genome. Nat. Genet. 39, 311-318 (2007).

31. Kellner, W. A., Ramos, E., Van Bortle, K., Takenaka, N. \& Corces, V. G. Genome-wide phosphoacetylation of histone H3 at Drosophila enhancers and promoters. Genome Res. 22, 1081-1088 (2012).

32. Kharchenko, P. V. et al. Comprehensive analysis of the chromatin landscape in Drosophila melanogaster. Nature 471, 480-485 (2011).

33. Negre, N. et al. A cis-regulatory map of the Drosophila genome. Nature 471, 527-531 (2011).

34. Dixon, J. R. et al. Topological domains in mammalian genomes identified by analysis of chromatin interactions. Nature 485, 376-380 (2012). 
35. Hou, C., Li, L., Qin, Z. S. \& Corces, V. G. Gene density, transcription, and insulators contribute to the partition of the Drosophila genome into physical domains. Mol. Cell 48, 471-484 (2012).

36. Lieberman-Aiden, E. et al. Comprehensive mapping of long-range interactions reveals folding principles of the human genome. Science 326, 289-293 (2009).

37. Nora, E. P. et al. Spatial partitioning of the regulatory landscape of the X-inactivation centre. Nature 485, 381-385 (2012).

38. Sexton, T. et al. Three-dimensional folding and functional organization principles of the Drosophila genome. Cell 148, 458-472 (2012).

39. Lin, C. Y. et al. Transcriptional amplification in tumor cells with elevated c-Myc. Cell 151, 56-67 (2012).

40. Nie, Z. et al. c-Myc Is a universal amplifier of expressed genes in lymphocytes and embryonic stem cells. Cell 151, 68-79 (2012).

41. Frye, M., Fisher, A. G. \& Watt, F. M. Epidermal stem cells are defined by global histone modifications that are altered by Myc-induced differentiation. PLoS ONE 2, e763 (2007).

42. Beck, D. B., Oda, H., Shen, S. S. \& Reinberg, D. PR-Set7 and H4K20me1: at the crossroads of genome integrity, cell cycle, chromosome condensation, and transcription. Genes Dev. 26, 325-337 (2012).

43. Kuo, A. J. et al. The BAH domain of ORC1 links H4K20me2 to DNA replication licensing and Meier-Gorlin syndrome. Nature 484, 115-119 (2012).

44. Driskell, I. et al. The histone methyltransferase Setd8 acts in concert with c-Myc and is required to maintain skin. EMBO J. 31, 616-629 (2012).

45. Ji, H. et al. Cell-type independent Myc target genes reveal a primordial signature involved in biomass accumulation. PLoS ONE 6, e26057 (2011).

46. Phillips, J. E. \& Corces, V. G. CTCF: master weaver of the genome. Cell 137, 1194-1211 (2009).

47. Chen, Q. et al. Recurrent trisomy and Robertsonian translocation of chromosome 14 in murine iPS cell lines. Chromosome Res. 19, 857-868 (2011).

48. Guffei, A. et al. c-Myc-dependent formation of Robertsonian translocation chromosomes in mouse cells. Neoplasia 9, 578-588 (2007).

49. Louis, S. F. et al. c-Myc induces chromosomal rearrangements through telomere and chromosome remodeling in the interphase nucleus. Proc. Natl Acad. Sci. USA 102, 9613-9618 (2005).
50. Wood, A. M. et al. Regulation of chromatin organization and inducible gene expression by a Drosophila insulator. Mol. Cell 44, 29-38 (2011).

51. Van Bortle, K. et al. Drosophila CTCF tandemly aligns with other insulator proteins at the borders of H3K27me3 domains. Genome Res. 22, 2176-2187 (2012).

52. Xu, H. et al. A signal-noise model for significance analysis of ChIP-seq with negative control. Bioinformatics 26, 1199-1204 (2010).

\section{Acknowledgements}

We thank members of the lab for helpful discussions and suggestions during this study. We also thank The Genomic Services Lab at the HudsonAlpha Institute for Biotechnology for their help in performing Illumina sequencing of ChIP-seq samples. Research reported in this publication was supported by the National Institute of General Medical Sciences of the National Institutes of Health under award number R01GM035463. The content is solely the responsibility of the authors and does not necessarily represent the official views of the National Institutes of Health.

\section{Author contributions}

V.G.C. planned the project; J.Y., P.G.D. and E.S. conducted experiments; J.Y. performed data analysis; J.Y. and V.G.C. wrote the manuscript and discussed the results.

\section{Additional information}

Accession codes: Sequence data have been deposited in NCBI's Gene Expression Omnibus (GEO) under accession numbers GSE32584 and GSE39521.

Supplementary Information accompanies this paper at http://www.nature.com/ naturecommunications

Competing financial interests: The authors declare no competing financial interests.

Reprints and permission information is available online at http://npg.nature.com/ reprintsandpermissions/

How to cite this article: Yang, J. et al. A subset of Drosophila Myc sites remain associated with mitotic chromosomes colocalized with insulator proteins. Nat. Commun. 4:1464 doi: $10.1038 /$ ncomms2469 (2013). 